\title{
Technical Vaccination Recommendations for COVID-19 Vaccines in China (First Edition)
}

\author{
COVID-19 Vaccine Technical Working Group
}

To date, five coronavirus disease 2019 (COVID-19) vaccines have been conditionally approved for market authorization or granted emergency use authorization in China by the National Medical Products Administration (NMPA). Three inactivated COVID-19 vaccines and one adenovirus-vectored vaccine were granted conditional approval, as phase III clinical trial data for these four conditionally-approved vaccines have demonstrated protective efficacy levels that meet requirements of NMPA's Guidelines for Clinical Evaluation of COVID-19 vaccine (interim version) and also meet requirements recommended by the World Health Organization (WHO) in their COVID-19 Vaccines Target Product Profile. Adverse event surveillance data from clinical trials, emergency use, and large-scale vaccination of key populations have demonstrated that the approved COVID-19 vaccines have excellent safety profiles. One recombinant subunit COVID-19 vaccine was approved for emergency use, as phase II clinical trial results showed good immunogenicity and safety of the recombinant subunit COVID-19 vaccine (CHO cell).

Based on clinical trial data of the NMPA-approved domestic vaccines and the epidemiological characteristics of COVID-19, the COVID-19 Vaccine Technical Working Group of China CDC drafted technical vaccination recommendations. The National Immunization Advisory Committee reviewed, discussed, and refined the recommendations. The National Health Commission accepted and published these recommendations (1).

These recommendations can be used by health departments and disease control institutions at all levels in China to guide COVID-19 vaccination.

\section{VACCINE TYPES}

\section{Inactivated COVID-19 Vaccines}

The three conditionally-approved inactivated COVID-19 vaccines are manufactured by Beijing
Institute of Biological Products Co., Ltd, Sinopharm (Beijing Institute), Wuhan Institute of Biological Products Co., Ltd, Sinopharm (Wuhan Institute), and Sinovac Research \& Development Co., Ltd (Sinovac). All three use Vero cells to culture and grow COVID-19 virus and $\beta$-propionolactone to inactivate the virus while retaining antigenic components that induce immune responses. All three are adjuvanted with aluminum hydroxide to improve immunogenicity.

\section{Adenovirus Vectored COVID-19 Vaccine}

The conditionally-approved adenovirus- 5 vectored COVID-19 vaccine is manufactured by CanSinoBIO (CanSino). This vaccine is made by recombining the spike-glycoprotein (S protein) gene of COVID-19 virus into the genetic material of a replication-deficient human adenovirus- 5 . After injection of the vaccine, the recombinant adenovirus brings the $S$-protein gene into the person's cells that then use the gene to make $S$ protein antigen, which induces an immune response in the person vaccinated.

\section{Recombinant Subunit COVID-19 Vaccine}

The recombinant subunit COVID-19 vaccine ( $\mathrm{CHO}$ cell) that has been approved for emergency use is manufactured by Anhui Zhifei Longcom Biopharmceutical Co., Ltd. This vaccine is made by recombining the $\mathrm{S}$-protein receptor binding domain (RBD) gene with a gene in Chinese hamster ovary $(\mathrm{CHO})$ cells. The $\mathrm{CHO}$ cells make $\mathrm{RBD}$ protein dimer that are purified and concentrated for use in producing the vaccine. Aluminum hydroxide adjuvant is added to improve immunogenicity.

\section{RECOMMENDED IMMUNIZATION SCHEDULE}

\section{Target Population}

The five COVID-19 vaccines approved for 
conditional marketing or emergency use are suitable for persons aged 18 years and above.

\section{Schedules}

Inactivated COVID-19 vaccines (Vero cell): Two doses are recommended with a minimum 3-week interval between doses. The second dose should be given at the earliest possible opportunity after 3 weeks, within 8 weeks.

Adenovirus vector COVID-19 vaccine (Ad5): One dose is recommended.

Recombinant subunit COVID-19 vaccine (CHO cell): Three doses are recommended with 4-week minimum intervals between doses. The second dose should be given at the earliest possible opportunity between 4 weeks and 8 weeks after the first dose. The third dose should be given within 6 months after the first dose, at least 4 weeks after the second dose.

\section{Route and Site}

All doses are recommended to be given by intramuscular injection in the deltoid muscle.

\section{SPECIAL CONSIDERATIONS}

\section{Delayed and Catch-Up Doses and Minimum Interval Violations}

Individuals who did not complete vaccination in the recommended time frame are recommended to receive the delayed dose(s) at the earliest possible opportunity. Delayed doses do not need to be repeated.

Individuals administered inactivated vaccine whose second dose was inadvertently administered less than 14 days after the first dose are recommended to be given one additional dose no earlier than 3 weeks after the second dose. Individuals administered inactivated vaccine who received a second dose between 14 and 21 days after the first dose do not need an additional dose.

\section{Booster Doses}

Currently, booster doses are not recommended.

\section{Co-administration with Other Vaccines}

At present, it is not recommended to administer COVID-19 vaccines with other vaccines. With the exception of rabies post-exposure vaccination and tetanus wound management, there should be a minimum interval of 14 days between administration of a COVID-19 vaccine and other vaccines. If rabies vaccine or tetanus toxoid needs to be administered due to animal bites, trauma, and any other emergency indication, there is no minimum interval following COVID-19 vaccination for administering rabies vaccine or tetanus toxoid.

\section{Interchangeability of COVID-19 Vaccines}

At present, it is suggested to use the same manufacturer's COVID-19 vaccine to complete a series once started. In special circumstances such as stockouts or changing vaccination sites, vaccines from other manufacturers that are made with the same technology platform can be used to complete the schedule.

\section{Infection and Antibody Tests}

Testing for COVID-19 virus infection with PCR or antibody tests prior to COVID-19 vaccination is not recommended. Routinely testing for antibodies after vaccination to determine protection is not recommended.

\section{Contraindications}

COVID-19 vaccination is contraindicated for (a) people who are allergic to any of the components or excipients in the vaccine or substances used in the production process, or who have had allergic reactions after vaccination with similar vaccines; (b) people with a history of severe allergic reaction to any vaccine (e.g., acute allergic shock, angioneurotic edema, dyspnea, or laryngeal edema, etc.); (c) people with uncontrolled epilepsy and other serious progressive neurological diseases (e.g., transverse myelitis, GBS, or demyelinating diseases, etc.); (d) people with fever, acute illness, acute exacerbation of a chronic disease, or uncontrolled severe chronic disease; and (e) pregnant women.

COVID-19 vaccine package inserts (prescribing instructions) specify precautions and contraindications for several specific groups of people and these specific population can be considered as follows.

\section{VACCINATION OF SPECIFIC POPULATIONS}

\section{Persons Aged 60 Years and Over}

The risk of severe disease and death due to COVID-19 is greatest in persons aged 60 years and 
over. The Phase III efficacy trials of the four conditionally approved COVID-19 vaccines had insufficient elderly subjects to make robust estimates of efficacy. Efficacy or effectiveness estimates for these vaccines are anticipated to be available in the future. Phase I / II clinical trial data for the five vaccines that have conditional approval or emergency use authorization showed that these vaccines were safe for this age group. Neutralizing antibody levels were slightly lower among elderly subjects than among subjects aged 18-59 year. However, seroconversion rates were similar, suggesting that these vaccines will be efficacious and effective in individuals aged 60 years and over. Therefore, these COVID-19 vaccines are recommended for use among people 60 years of age and older.

\section{Children and Adolescents Below the Age of 18 Years}

There are currently no efficacy or safety data for children or adolescents below the age of 18 years. Until such data are available, vaccination for individuals below 18 years of age is not recommended.

\section{Persons with Comorbidities}

Certain comorbidities have been identified as increasing the risk of severe COVID-19 disease and death. Vaccination is recommended for persons with comorbidities who are healthy or have conditions that are controlled well with medicine.

\section{Childbearing Age and Lactating Women}

Although these vaccines are currently contraindicated in pregnancy due to lack of clinical data and experience, they are anticipated to be safe for use in pregnancy. Pregnant women, at any stage of pregnancy, who are inadvertently vaccinated should not take any special action, including termination, because of the vaccination. Women who become pregnant after vaccination also should not take any specific action. Routine physical examinations during pregnancy and follow-up are recommended. Women planning pregnancy after getting vaccinated do not need to delay pregnancy.

Although there are currently no data about the impact of COVID-19 vaccine on breastfeeding child, on the basis of the safety profile of the above vaccines, a lactating woman who is otherwise recommended for vaccination, such as healthcare workers, should be offered vaccination. In reference to international practices, breastfeeding after vaccination is recommended as routine.

\section{Immunocompromised Persons}

Immunocompromised persons are at higher risk of severe disease and death due to COVID-19. Available data are currently insufficient to assess vaccine efficacy or safety in immunocompromised persons (such as persons with malignancies, nephrotic syndrome, and AIDS) and persons with HIV infection. It is possible that the immune response to vaccination may be reduced, lowering clinical effectiveness. Given the safety profiles of similar types of vaccines, inactivated COVID-19 vaccines and recombinant subunit vaccines are recommended for use. Regarding the adenovirus vectored COVID-19 vaccine, although the adenovirus is replication defective, there are no safety data for similar vaccines in this population. Immunocompromised persons may receive adenovirus vectored COVID-19 vaccine after assessing benefits and risks of the vaccine with the help of a health care provider.

\section{Persons Who Have Previously Had COVID-19}

Currently available data indicate that symptomatic reinfection within the first six months after an initial infection is rare. Persons who have previously had COVID-19 (symptomatic or asymptomatic) may receive one dose of any of the COVID-19 vaccines at least six months after infection.

\section{OTHER CONSIDERATIONS}

These recommendations will be updated upon approval of additional COVID-19 vaccines as more information is available from clinical trials, postmarketing surveillance, and evaluations, and as changes in the COVID-19 epidemiological situation warrant.

doi: $10.46234 / \mathrm{ccdcw} 2021.083$

Submitted: March 29, 2021; Accepted: March 30, 2021

\section{REFERENCES}

\footnotetext{
1. National Health Commission of the People's Republic of China. Technical vaccination recommendations for COVID-19 vaccines in China (First Edition). http://www.nhc.gov.cn/jkj/s3582/202103/ c2febfd04fc5498f916b1be080905771.shtml?R0NMKk6uozOC=161708 8446795. [2021-03-29]. (In Chinese).
} 